\title{
EFECTOS DEL CAMBIO CLIMÁTICO SOBRE LA DISTRIBUCIÓN POTENCIAL DE LOS FLEBOTOMINOS TRANSMISORES DE LEISHMANIASIS EN MÉRIDA VENEZUELA
}

\author{
EFFECTS OF CLIMATE CHANGE ON THE POTENTIAL DISTRIBUTION OF SANDFLIES \\ TRANSMITTERS OF LEISHMANIASIS IN MERIDA VENEZUELA
}

\author{
Elsa Nieves, Maria Rujano, Héctor Ospino, Luzmary Oraá, Yorfer Rondón, Mireya Sánchez, Maritza Rondón, \\ Yetsenia Sánchez, Masyelly Rojas, Nestor González y Dalmiro Cazorla
}

\begin{abstract}
RESUMEN
La leishmaniasis constituye un grave problema de salud pública en diferentes partes del mundo. Es conocido que los efectos del calentamiento global han aumentado en los últimos años, con la consecuente modificación, a corto y largo plazo, de los hábitats de los flebotominos, lo que potencialmente puede impactar en la transmisión de la leishmaniasis. En el presente trabajo se evaluó el efecto del cambio climático en la distribución geográfica de los flebotominos transmisores de la leishmaniasis cutánea, en Mérida, Venezuela. Para el análisis se tuvieron en cuenta cuatro especies vectoras Lutzomyia youngi, L. gomezi, L. ovallesi y L. walkeri y se usó un modelo atmósfera-océano Hadcm3, bajo la premisa de cambio climático en un escenario pesimista A2, con proyecciones para los años 2020, 2050 y 2080. Los resultados del modelo mostraron para L. youngi un incremento en su distribución, para L. gomezi cambios y desplazamiento en su distribución potencial futura, para L. ovallesi se mostró como la especie que puede ser más afectada por el aumento de la temperatura, con predicciones de cambios en su distribución futura, y para la distribución de L. walkeri no se mostró afectación por el cambio climático. Por otro lado, el modelo recreó escenarios de cambio climático, con predicciones de solapamiento de las principales especies transmisora, en los municipios de mayor densidad poblacional, lo que sugiere un mayor riesgo de transmisión de la leishmaniasis en el estado Mérida en los próximos años. Los mapas generados proporcionan información relevante para alertar sobre cambios en los patrones de transmisión de la leishmaniasis, además serán una herramienta útil para los entes de control y prevención.
\end{abstract}

PALABRAS CLAVE: Cambio Climático, Lutzomyia; leishmaniasis, MaxEnt; distribución potencial

\begin{abstract}
Leishmaniasis is a serious public health problem in different parts of the world. It is known that the effects of climate change has been increasing in recent years by habitat changing of sandflies the short and long term what can impact the transmission of leishmaniasis. In the current work evaluates the effect of climate change on the geographical distribution of sandflies transmitters of cutaneous leishmaniasis in Merida Venezuela. The analysis includes 4 species, Lutzomyia youngi, L. gomezi, L. ovallesi and L. walkeri using an atmosphere-ocean model HadCM3 under the premise of climate change in a pessimistic scenario A2 with projections for 2020, 2050 and 2080. Results predicted an increased in distribution of L. youngi, while for L. gomezi changes in its future potential distribution. L. ovallesi was the species most affected presented changes at future distribution and distribution of $L$. walkeri not affected by climate change. In addition, the model in a climate change scenario predicts an overlap of the main transmitter species in the municipalities of greater population density, suggesting an increased risk of transmission of leishmaniasis in Merida state in the coming years, the maps generated provide information relevant to understanding of the transmission of leishmaniasis and to authorities in control and prevention.
\end{abstract}

KEY WORDS: Distribution; Climate change; Lutzomyia; Leishmaniasis; MaxEnt; Sandflies

60

Dirección de los autores:

Laboratorio de Parasitología Experimental (LAPEX), Departamento de Biología, Facultad de Ciencias, Universidad de Los Andes, 5101 Mérida, Venezuela. Autor de correspondencia: nievesbelsa@gmail.com (E.N.), (M.R.), (L.O.), (Y.R.), (M.S.), (M.R.), (Y.S.), (M.R.), (N.G.). Laboratorio de Sostenibilidad y Ecodiseño, Facultad de Ciencias Forestales y Ambientales, Facultad de Arquitectura y Diseño Universidad de Los Andes, 5101 Mérida, Venezuela (M.R.), (H.O.). Laboratorio de Entomología, Parasitología y Medicina Tropical (LEPAMET), Universidad Nacional Experimental Francisco de Miranda, 4101 Falcón, Venezuela (D.C.). 


\section{INTRODUCCIÓN}

El parásito Leishmania Ross, 1903, causante de la leishmaniasis es transmitido mediante la picada de varias especies de insectos hematófagos pertenecientes al género Phlebotomus Rondani y Berte, 1840, en el viejo mundo y del género Lutzomyia França, 1924, en el nuevo mundo (Alexander y Maroli, 2003; Neuber, 2008). El cambio climático global contribuye ampliar la distribución de patógenos transmitidos por vectores en el tiempo y el espacio, exponiendo así a las poblaciones a estaciones de transmisión más larga (Reiter, 2001; Kilpatrick et al., 2010; Moo et al., 2013). En la actualidad, es un hecho científico que el clima global se ha alterado significativamente, los estudios muestran que la temperatura anual del planeta se va incrementando (Shaw, 2007), la modificación de la temperatura promedio podría tener efectos a corto y largo plazo sobre los hábitats de los vectores, incrementando el número de criaderos, distribución y densidad de vectores (Reiter, 2001; Kigadye et al., 2010; Fischer, 2011). Durante las últimas décadas, la incidencia de la leishmaniasis cutánea a escala mundial, ha aumentado a un ritmo alarmante, como consecuencia del cambio climático, el uso de la tierra, la migración y los patrones de urbanización, así como a otros factores (Campbell et al., 2001; Desjeux, 2001; Loiola et al., 2007; Salomón et al., 2008; OMS, 2010; Parra, 2010; Alvar et al., 2012; Vásquez et al., 2013).

La leishmaniasis presenta una amplia distribución geográfica en Venezuela; se han registrado casos en 23 de las 24 entidades federales, la región Andina venezolana es un área de alta endemicidad para la leishmaniasis cutánea (Moreno y Scorza, 1998; Rodríguez et al., 2007; Nieves et al., 2014a), en el estado Mérida la zona del Valle del Mocotíes, es la que presentan mayor número de casos (Valera et al., 1978; Scorza et al., 1983; 1985; Maingon et al., 1994; Lugo et al., 1999, 2003; Rodríguez et al., 2007, 2010; Nieves et al., 2015). Oraá (2013), registró en el estado Mérida a L. youngi, L. gomezi, $L$. ovallesi y L. walkeri, como las especies más abundantes y que presentaron infección natural por Leishmania, perteneciente a los sub-géneros Leishmania y Viannia.

Los modelos predictivos son una herramienta importante en una variedad de aplicaciones en ecología y conservación (Graham et al., 2006; Blanco, 2013) y de cambio climático (Bässleret et al., 2009). Los Sistemas de Información Geográfica, permiten integrar variables ecológicas asociadas a las especies y así entender las variables que permiten predecir su distribución (Peterson, 2006). Con estos métodos se desarrollan mapas predictivos para conocer nuevas áreas de distribución de enfermedades bajo diferentes presiones (Peterson y Robins, 2003).

Varios investigadores han documentado, mediante modelado matemático, que un aumento en la temperatura puede resultar en una expansión de la distribución geográfica y aumento de las abundancias de las especies de flebotominos, con el subsiguiente aumento de casos de leishmaniasis (Cross y Hyams, 1996; Thomson et al., 1999; Magnuson, 2001; González et al., 2010; Fischer et al., 2011; Hartemink et al., 2011). En el presente trabajo se evaluó la distribución geográfica de los vectores de leishmaniasis cutánea, en Mérida Venezuela, con la utilización de un modelo matemático bajo escenarios de cambio climático, para los años 2020, 2050 y 2080, con el fin de predecir los lugares de distribución potencial de las especies y los posible riesgo de transmisión.

\section{MATERIALES Y MÉTODOS}

\section{Área de estudio}

Los flebotominos se capturaron en 21 de los 23 municipios del Estado Mérida, en un gradiente altitudinal entre 61 y 2099 m. El Estado Mérida se localiza en el occidente de Venezuela entre los $07^{\circ} 34^{\prime} 60.0^{\prime \prime}, 09^{\circ} 21^{\prime} 0.0^{\prime \prime}$ de latitud Norte y los $70^{\circ} 30^{\prime} 0.0^{\prime \prime}, 72^{\circ} 0^{\prime} 0.0^{\prime \prime}$ de longitud Oeste, Figura 1. Debido a las variaciones altitudinales que presenta, tiene una variación climática que va desde los climas semiáridos en las partes más bajas; hasta los climas de páramo. Las múltiples combinaciones de estos factores, altitud, temperatura y precipitación, a su vez condicionan la existencia de una gran variedad de tipos de vegetación, que abarcan diferentes zonas (Ataroff y Sarmiento, 2004).

\section{Datos Entomológicos}

Los flebotominos se capturaron utilizando cuatro método, con trampas de Shannon, trampas adhesivas, trampas CDC y aspiración directa como lo describe Nieves et al. (2014b). Se realizaron dos capturas semanales entre mayo de 2012 y mayo de 2014. La identificación de los flebotominos se realizó mediante morfología externa e interna según los criterios de Young y Duncan (1994). Los puntos de captura fueron georreferenciados mediante un equipo GPS-Garmin- 
Oregón 550. Los datos del análisis incluyeron 190 puntos de ocurrencia georreferenciados entre las cuatro especies más abundantes capturadas e incriminadas en la transmisión de la leishmaniasis, Lutzomyia youngi Feliciangeli y Murillo, 1985 (67 puntos de ocurrencia), Lutzomyia gomezi Nitzulescu, 1931 (51 puntos de ocurrencia), Lutzomyia ovallesi Ortiz, 1952 (40 puntos de ocurrencia) y Lutzomyia walkeri Newstead, 1914 (32 puntos de ocurrencia). Todos por encima del valor mínimo de más de 30 registros por especie, para obtener modelos estadísticamente válidos (Wisz et al., 2008).

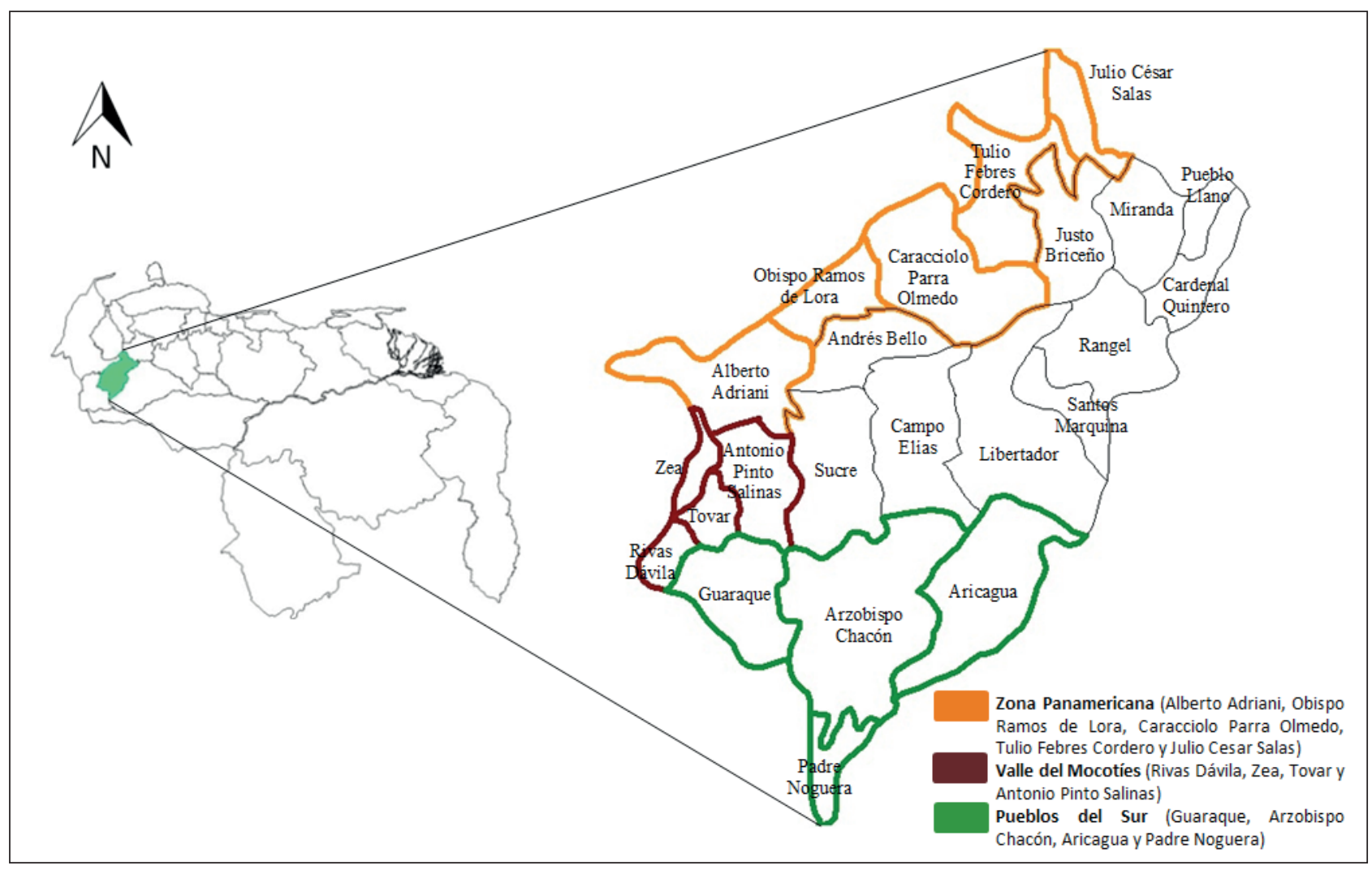

Figura 1. Ubicación geográfica del Estado Mérida-Venezuela, con sus municipios y principales zonas.

\section{Datos climáticos}

Se usaron 19 variables bioclimáticas que corresponden a capas ambientales en formato raster para el estado Mérida, derivadas de la interpolación de datos medios mensuales de precipitación y temperatura registrados en estaciones meteorológicas desde 1950 al 2000, disponibles en la base de datos Worldclim (http://www.worldclim.org), para la zona 23 con una resolución de 30 arco segundos $(1 \mathrm{~km})$ (Hijmans 62 et al., 2005a). Estas variables fueron: 1) temperatura promedio anual $\left({ }^{\circ} \mathrm{C}\right) ; 2$ ) rango diurno de temperatura $\left({ }^{\circ} \mathrm{C}\right)$; 3) isotermalidad $\left({ }^{\circ} \mathrm{C}\right)$; 4) estacionalidad de temperatura (\%);5) temperatura máxima del período más caliente $\left({ }^{\circ} \mathrm{C}\right)$; 6) temperatura mínima del período más frío $\left({ }^{\circ} \mathrm{C}\right)$; 7) rango anual de temperatura $\left({ }^{\circ} \mathrm{C}\right)$; $8)$ temperatura media en el trimestre más lluvioso $\left({ }^{\circ} \mathrm{C}\right)$; 9) temperatura promedio en el trimestre más seco $\left.\left({ }^{\circ} \mathrm{C}\right) ; 10\right)$ temperatura promedio en el trimestre más caluroso $\left({ }^{\circ} \mathrm{C}\right)$; 11$)$ temperatura promedio en el trimestre más frío $\left({ }^{\circ} \mathrm{C}\right)$; 12) precipitación anual (mm); 13) precipitación en el período más lluvioso $(\mathrm{mm})$; 14) precipitación en el período más seco $(\mathrm{mm})$; 15) estacionalidad de la precipitación (\%); 16) precipitación en el trimestre más lluvioso $(\mathrm{mm})$; 17) precipitación en el trimestre más seco $(\mathrm{mm})$; 18$)$ precipitación en el trimestre más caluroso (mm) y 19) precipitación en el trimestre más frío $(\mathrm{mm})$. 


\section{Distribución potencial de flebotominos bajo escenarios de cambio climático}

Para determinar el efecto del cambio climático sobre la distribución potencial de las especies transmisoras de leishmaniasis en Mérida, se empleó el modelo matemático de circulación global Hadcm3 (Hadley Centre Coupled Model, por sus siglas en Inglés), disponible en http://www.ccafs-climate.org/. El Hadcm3 es un modelo atmósfera-océano que se encuentra ampliamente descrito por Gordon et al. (2000) y Pope et al. (2000). Se trata de un modelo reciente, con características aceptables (Stott et al., 2001), que puede utilizarse para escenarios pesimista A2 y optimista B2, en conjunción con variables bioclimáticas (Delgado y Suarez, 2009; Gaertner et al., 2012). Para este estudio se seleccionó el escenario pesimista A2, que se describe para los años 2020, 2050 y 2080, el cual corresponde a un mundo muy heterogéneo, que proyecta un aumento continuo de las emisiones globales de gases de efecto invernadero, lo que resulta en un incremento de la temperatura y una disminución sustancial de la lluvia en la mayor parte de Suramérica. Sus características más distintivas son la autosuficiencia y la conservación de las identidades locales. Este escenario A2, supone un aumento de la población, de 15.000 millones de habitantes para el año 2100. El desarrollo económico está basado principalmente en las regiones y un crecimiento económico por habitante, así como el cambio más fragmentados y menos impactantes que en otras líneas de desarrollo (Honty, 2011; Moo et al., 2013).

Para el modelado matemático actual y futuro se utilizó MaxEnt versión 3.3 (Phillips et al., 2006), basado en el análisis de máxima entropía (Phillips y Dudik, 2008), con los valores predeterminados del programa y el umbral de corte (10 Percentile Training Presence), para maximizar la sensibilidad y minimizar la especificidad (Phillips et al., 2006). El umbral se interpreta como el $90 \%$ de los puntos de presencia se encuentran dentro del área potencial, donde las condiciones climáticas son apropiadas para la ocurrencia de la especie, mientras que el $10 \%$ restante de los puntos que caen fuera del área potencial con un ambiente atípico. Entre más alto es el umbral, existe mayor restricción del área de distribución de las especies (Scheldeman y van Zonneveld, 2011; Valera et al., 2014). MaxEnt modela el nicho fundamental de las especies mediante la relación entre un conjunto de localidades (puntos de presencia), donde se conoce que la especie está presente y un grupo de variables bioclimáticas que limitan la capacidad de supervivencia de la especie. Así mismo, los modelos predictivos actuales y futuros fueron representados, a través del programa de Sistema de Información Geográfica, Diva GIS versión 7.5 (Hijmans et al., 2005b), para obtener un mapa final idóneo al hábitat por cada especie (Anderson et al., 2003; Wang et al., 2010).

El modelo matemático de distribución actual representa un rango de 0-umbral (color blanco) indicando las áreas donde el modelo coincide que la especie está ausente; de umbral-0.3 (color verde) las áreas con baja probabilidad de presencia; de 0.3-0.5 (color amarillo), las áreas con probabilidad media de presencia; de 0.50.7 (color naranja) las áreas con alta probabilidad de presencia y de 0.7-1 (color rojo) las áreas en donde los modelos predicen que las condiciones climáticas son excelentes para la presencia de las especies (Anderson et al., 2003).

Los modelos matemáticos predictivos futuros, están representados en cuatros áreas: de color blanco, áreas por fuera del nicho realizado que no son adecuadas ni con las condiciones actuales ni con las condiciones futuras; de color rojo, áreas de alto impacto en que la especie probablemente ocurra en las condiciones climáticas actuales pero que dejarán de ser adecuadas en el futuro; de color azul, áreas de bajo impacto en que la especie probablemente ocurra tanto en las condiciones climáticas actuales como en las condiciones climáticas futuras; de color verde, nuevas áreas adecuadas en que una especie tendría probabilidades de ocurrir en el futuro, pero que no son idóneas para la ocurrencia natural en las condiciones actuales (Scheldeman y van Zonneveld, 2011).

\section{RESULTADOS}

El umbral que limita el área de distribución actual de L. youngi fue de 0,395 y bajo el escenario de cambio climático de 0,257 , este resultado muestra el efecto del cambio climático sobre el área de distribución de la especie, por el cual se favorecerá su distribución potencial en el futuro, en zonas que no son idóneas para la ocurrencia natural en las condiciones actuales (Figura 2). La expansión de su distribución se dará hacia áreas de la zona Panamericana, del Valle del Mocotíes y zonas de los municipios Campo Elías y Libertador. Para la proyección en el año 2080, ganaría nuevos espacios favorables en la zona Panamericana, en el municipio Campo Elías, Libertador y en gran parte de los municipios de los Pueblos del Sur. 


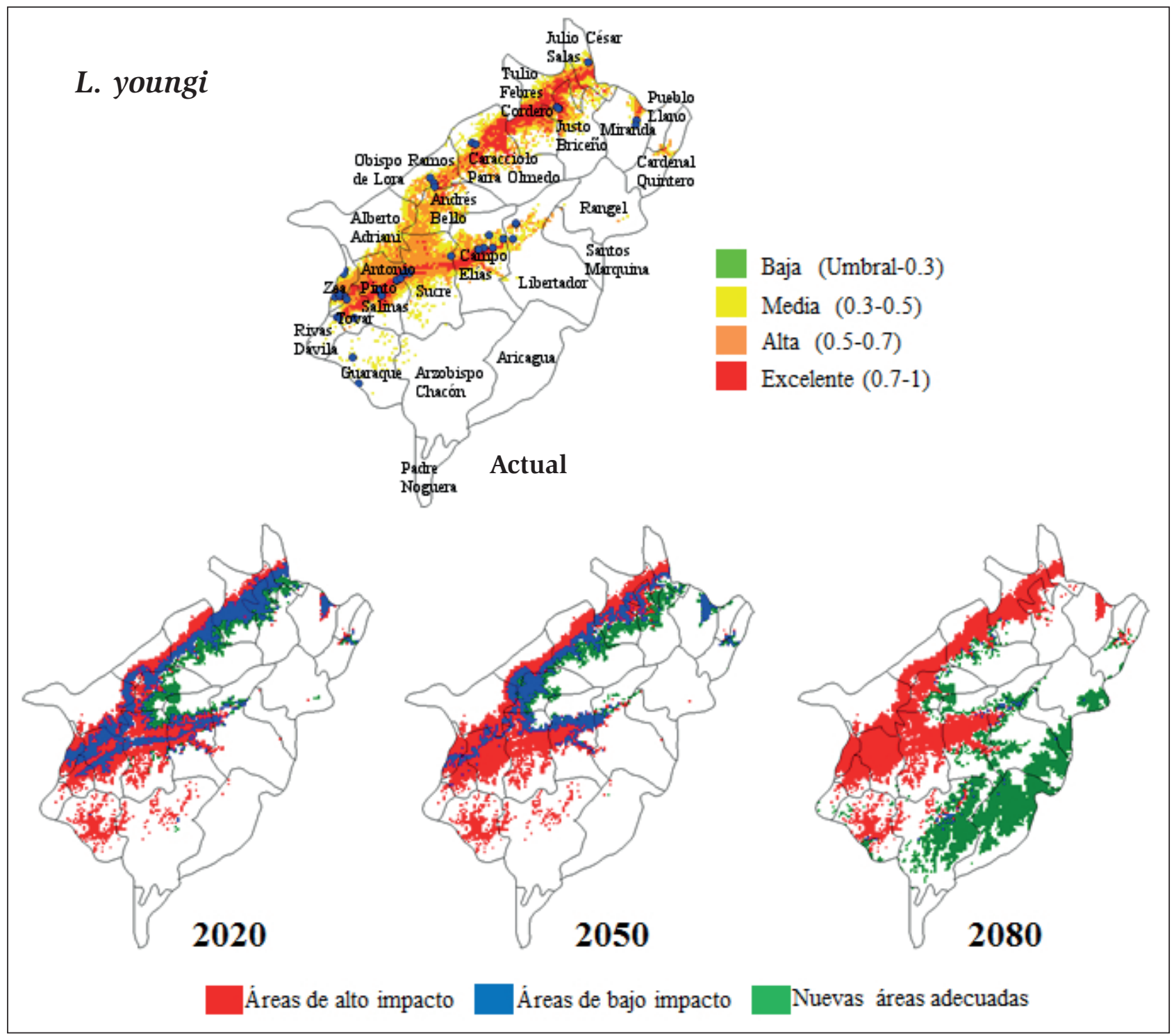

Figura 2. Mapa de distribución actual y bajo escenarios de cambio climático de L. youngi para los años 2020 , 2050 y 2080 en el estado Mérida Venezuela. La predicción futura se realizó con el modelo matemático Hadcm3 en el escenario pesimista A2, utilizando las capas de 19 variables bioclimáticas derivadas de temperatura y precipitación.

El umbral para L. gomezi, mostró una restricción del área de distribución en el futuro, el umbral de distribución actual fue menor de 0,097 , con relación al escenario de cambio climático que fue de 0,145 , se muestra una pequeña disminución en su distribución en la zona Panamericana y áreas del Valle del Mocotíes, para la proyección para el año 2020, para el año 2050 esa distribución se mantiene y gana espacio en pequeñas zonas de la zona Panamericana y de los municipios más poblados, mientras que en el año 2080 se consolidaría su distribución a áreas bajas y calientes de la zona Panamericana y del Valle del Mocotíes (Figura 3).

El umbral que limita el área de distribución actual de L. ovallesi fue de 0,515 y bajo el escenario de cambio climático de 0,493. Estos valores similares indican que el área de distribución potencial de la especie, en escenario de cambio climático, tendría poca expansión en su área de distribución futura (Figura 4). Esta especie es la más susceptible en el futuro a cambios 
climáticos, con una disminución de su distribución para el año 2020, las proyecciones para el año 2050 la distribución en las áreas actuales se va a modificar extendiéndose a nuevas áreas en la zona Panamericana e incluso en zonas de los municipios de Sucre, Campo Elías y Libertador y para el año 2080 la distribución estará restringida a nuevas áreas de expansión de los Pueblos del Sur.

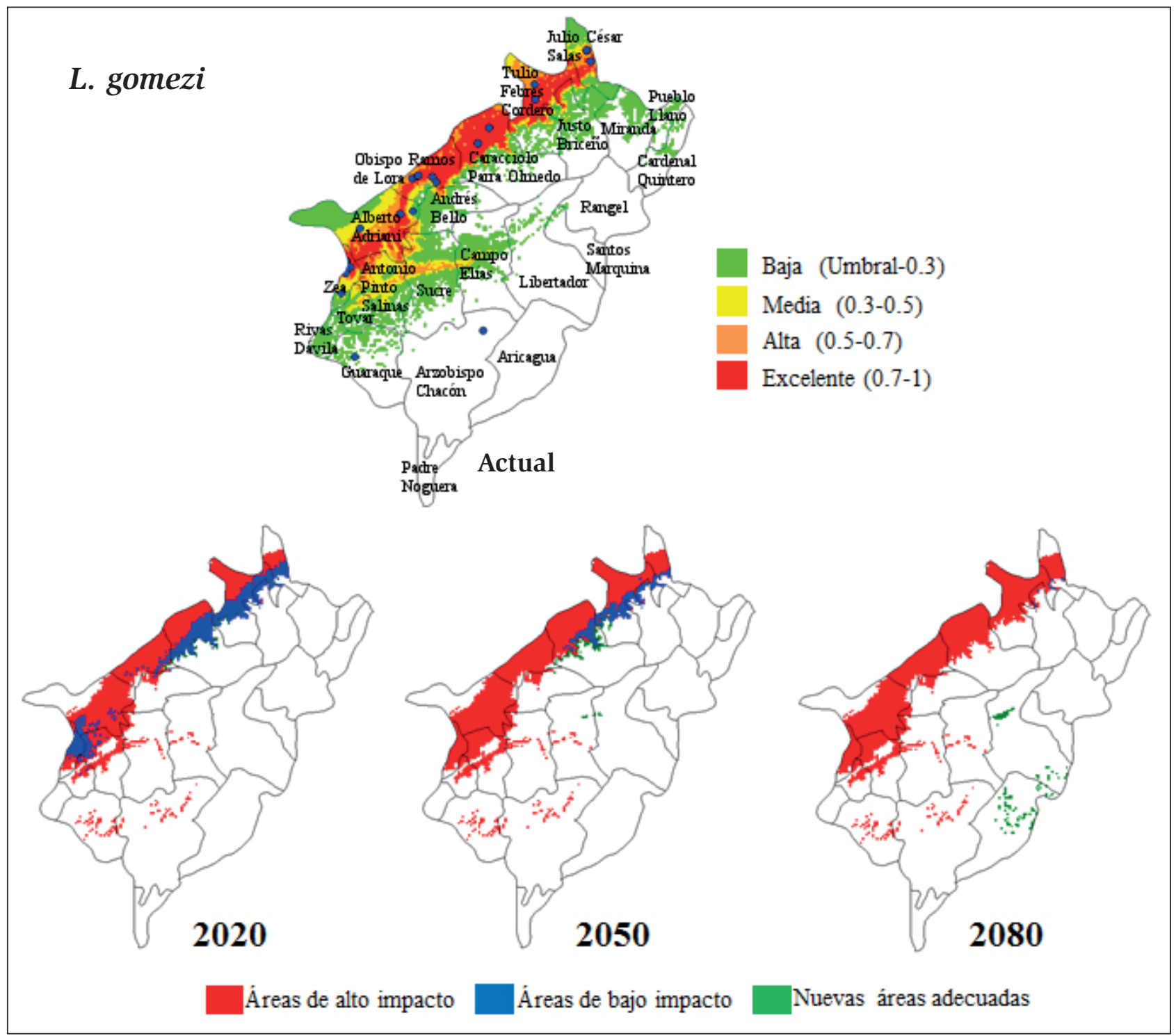

Figura 3. Mapa de distribución actual y bajo escenarios de cambio climático de L. gomezi para los años 2020, 2050 y 2080 en el estado Mérida Venezuela. La predicción futura se realizó con el modelo matemático Hadcm3 en el escenario pesimista A2, utilizando las capas de 19 variables bioclimáticas derivadas de temperatura y precipitación. 


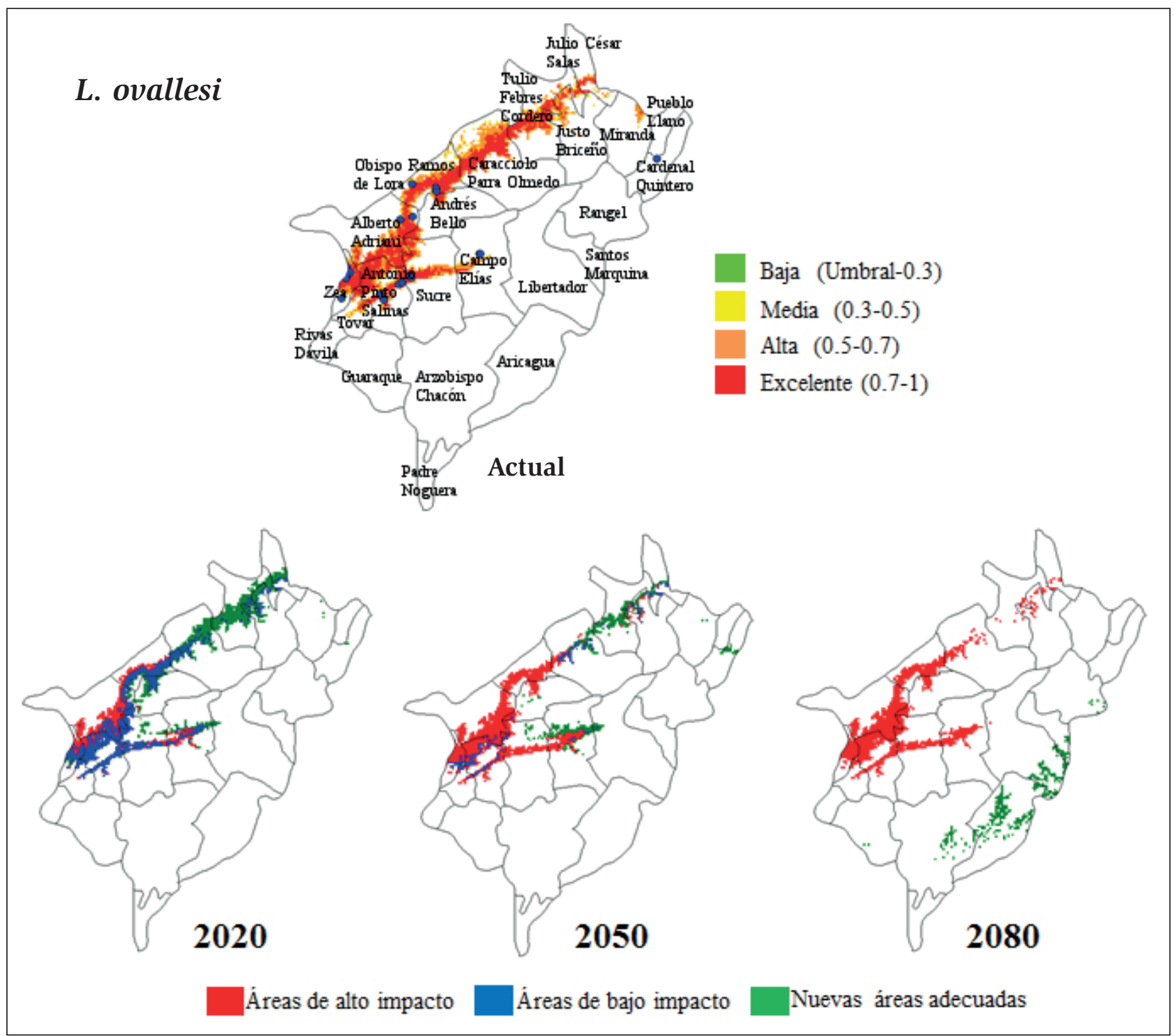

Figura 4. Mapa de distribución actual y bajo escenarios de cambio climático de L. ovallesi para los años 2020 , 2050 y 2080 en el estado Mérida Venezuela. La predicción futura se realizó con el modelo matemático Hadcm3 en el escenario pesimista A2, utilizando las capas de 19 variables bioclimáticas derivadas de temperatura y precipitación.

El umbral que limita el área de distribución actual y bajo el escenario de cambio climático para L. walkeri mostró valores similares de 0,409 , por lo cual se entiende que la distribución potencial de la especies bajo escenario de cambio climático se mantendrá igual a la actual para los años 2020 y 2050 y con la ocupación de nuevas áreas en el municipio Libertador para el año 2080 (Figura 5). 


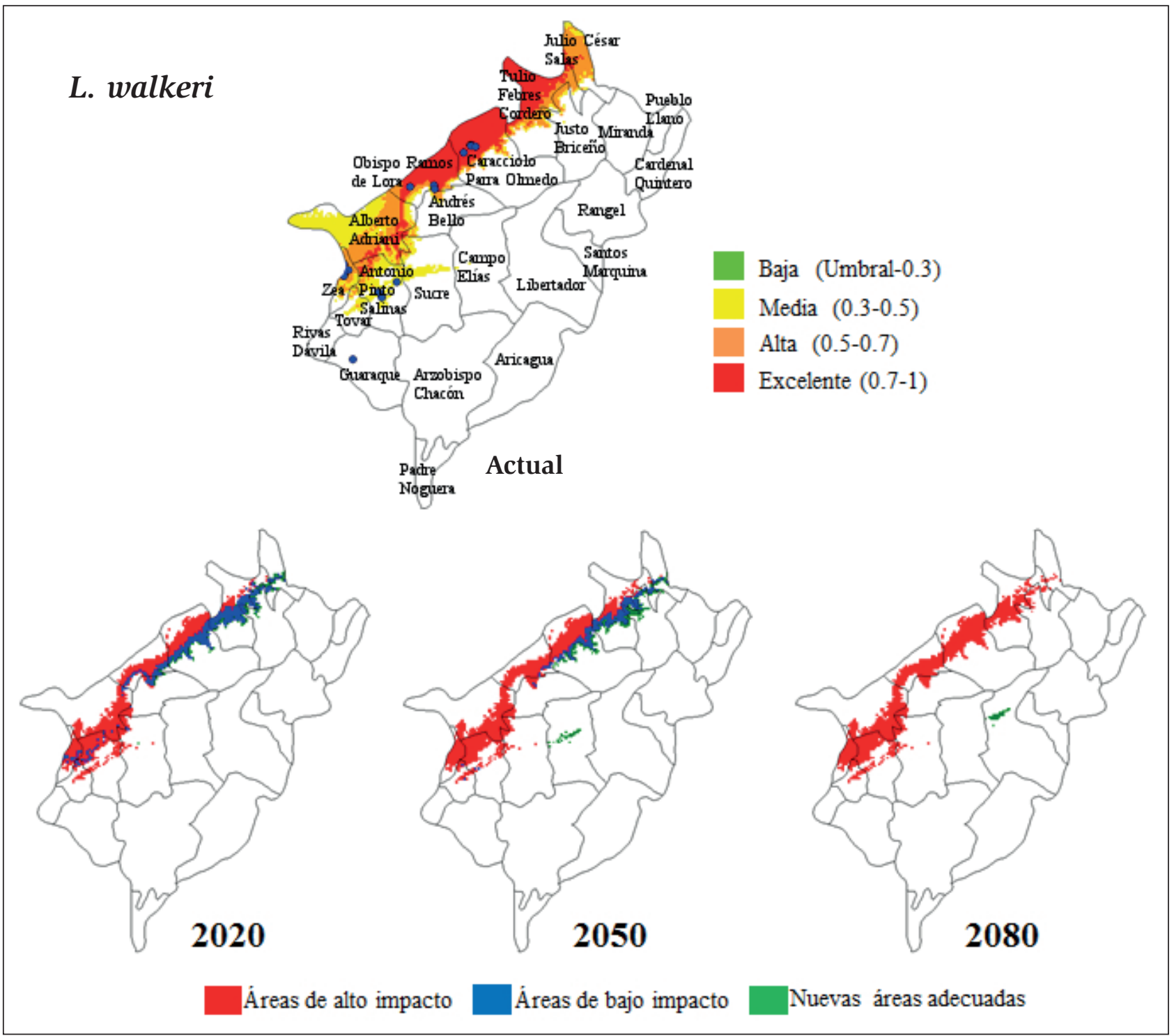

Figura 5. Mapa de distribución actual y bajo escenarios de cambio climático de L. walkeri para los años 2020 , 2050 y 2080 en el estado Mérida Venezuela. La predicción futura se realizó con el modelo matemático Hadcm3 en el escenario pesimista A2, utilizando las capas de 19 variables bioclimáticas derivadas de temperatura y precipitación.

\section{DISCUSIÓN}

Profundos cambios ocasionados por la destrucción de los ecosistemas por fenómenos naturales o producidos por los humanos, tales como, inundaciones, deforestaciones, cambios en los patrones de la vegetación, ha causado que los ciclos de transmisión de las leishmaniasis tengan igual impacto; mientras unas especies de flebotominos desaparecen otras se tornan más abundantes o se adaptan a los ambientes sinantrópicos, modificando su conducta (Días et al., 2003; Peterson y Shaw, 2003; Nieves et al., 2014b). Recientemente, la velocidad con la que ocurre el incremento de la temperatura, ha generado riesgos biológicos nunca antes vistos (Kuhn, 1999; Fischer et al., 2011). Los resultados del presente trabajo son realmente alarmantes ya que el cambio climático influiría en la distribución de los flebotominos en el estado Mérida, con un posible incremento en el riesgo de transmisión de la leishmaniasis. Aunque, Rodríguez et al. (2007), sugieren que los cambios en las condiciones ecológicas y climáticas, ocurridos en los últimos años en Mérida, debido a fenómenos como la vaguada y el consecuente deslave, ocurrido en las zonas adyacentes a la cuenca del río Mocotíes, pueden 
haber afectado la ocurrencia de casos de leishmaniasis, ya que se presentó una disminución de casos en los últimos años, este hecho pudo deberse a la disminución de transmisores, diezmados por la alteración de las condiciones ambientales, tal como ocurre, después del fenómeno de La Niña, donde se ha observado una disminución de enfermedades transmitidas por vectores (Cabaniel et al., 2005; Pabón y Nicholls, 2005; Cárdenas et al., 2006).

Por el contrario, las proyecciones a futuro bajo escenarios de cambio climático, con un incremento de la temperatura, muestran que para los años 2020, 2050 y 2080, que tendrían un efecto individual por cada especie transmisora. Así, como cada especie tiene un particular impacto en la transmisión de la leishmaniasis (Donalisio et al., 2012), por ejemplo L. youngi presentaría, en el futuro inmediato, un aumento en su distribución. Previa evaluación sobre la flebotomofauna en el estado Mérida, se registraron 35 especies de Lutzomyia, de las cuales 10 son consideradas antropofílicas y L. youngi considerada la principal especie transmisora y con mayor abundancia en localidades altas del estado, ubicadas entre los 800 y 1000 m (Rondón, 2015).

Los resultados predicen que $L$. youngi ganaría nuevos espacios de mayor altitud en el estado Mérida, producto al cambio climático, lo cual podría aumentar el riesgo de transmisión. Este hecho concuerda con modelos matemáticos que predicen que un aumento en la temperatura produciría un incremento en la abundancia de Phlebotomus perfiliewi, en toda Italia y la posibilidad de extender su distribución hacia el sur y el norte de Europa (Fischer et al., 2011). Igualmente se predice que Phlebotomus perniciosus Newstead, 1911 se extendería hacia Suiza en un ambiente más cálido (López y Molina, 2005). También, se ha previsto que en un aumento de la temperatura entre $1-5{ }^{\circ} \mathrm{C}$, pudiesen provocar la expansión de la distribución geográfica de Phlebotomus papatasi Scopoli, 1786, al suroeste de Asia (Cross et al., 1996), incluso una expansión en la distribución para las especies de Lutzomyia anthophora Addis, 1945, Lutzomyia diabólica Hall, 1936, Lutzomyia shannoni Dyar, 1929 y Lutzomyia cruciata Coquillett, 1907 en México y Estados Unidos (González et al., 2010; Moo et al., 2013; Peterson y Shaw, 2003).

Por otro lado, Rondón (2015) registró a L. gomezi, como la principal especie transmisora y predominante en los municipios de la zona Panamericana, que corresponde a los municipios con menor altura y mayor temperatura.
Los resultados sobre cambio climático predicen en un futuro inmediato que el aumento de temperatura provocaría la pérdida de espacios para L. gomezi en la zona Panamericana y se desplazaría hacia zonas más adecuadas del Valle del Mocotíes, con la ocupación de nuevos espacios en los municipios de los Pueblos del Sur y Libertador. El cambio en la distribución implica que ésta especie podría tener la capacidad de adaptarse o de migrar. La adaptación dependerá de la conservación de la vegetación y plasticidad genética de esta especie (Peterson y Shaw, 2003; Moo et al., 2013). Recientemente, Valderrama et al. (2011), registró que $L$. gomezi es la principal especie transmisora en Panamá y presenta una gran variabilidad genómica, con gran potencial de colonización, que le confiere la capacidad de migrar o adaptarse a cambios del medio ambiente. Además, L. gomezi presenta una amplia distribución en Venezuela, con el modelo de nicho ecológico, se muestra una distribución ideal hacia la zona noroccidental del país con varias coberturas vegetales y amplio rango de altura (Sánchez et al., 2015).

Por su parte, L. ovallesi se mostró como la especie más vulnerable al cambio climático. Aunque Sánchez et al. (2015), clasifica a la especie como propia de la región de los Andes Venezolanos, con una distribución potencial hacia la zona noroccidental y oriental de Venezuela. En un futuro inmediato esta especie, restringiría su distribución hacia el Valle del Mocotíes y para 2050 retomaría espacios de los municipios de la zona Panamericana, Campo Elías, Libertador y municipios de tradición turística, como Santo Domingo y para 2080 ocuparía nuevas zonas de los Pueblos del Sur. González et al. (2014) registraron una reducción de la distribución espacial, en las proyecciones de cambio climático de transmisores de leishmaniasis visceral, de Lutzomyia longipalpis y Lutzomyia evansi en Colombia. Esto podría deberse a diferencias genéticas presentes en las especies, a su adaptación y la capacidad de adecuación a los aumentos de temperatura.

Aunque, Feliciangeli (1987) registró que L. gomezi es afectada por la temperatura y L. ovallesi por la precipitación, lo que podría explicar, que cada entorno geográfico tiene sus propias características y que la distribución de una especie en un entorno depende de una serie de factores. A esto se le suma registros contradictorios con relación al efecto de la altitud en la distribución potencial de los flebotominos (Moo et al., 2013). A su vez, el cambio climático genera además, del incremento en la temperatura, cambios en los patrones 
de precipitaciones, alteraciones en el uso del suelo, cobertura vegetal y eventos bioclimáticos que pueden originar adaptaciones particulares entre las especies (Fischer et al., 2011; González et al., 2014).

Por otro lado, para la especie L. walkeri, el efecto del cambio climático producirá leves cambios en su distribución para el año 2080, que podría originar nuevos espacios de distribución en el municipio más poblado del Libertador. Si bien L. walkeri es la cuarta especie en orden de abundancia para las zonas bajas del estado Mérida, Añez et al. (1988) y Feliciangeli et al. (1988), la refieren como una especie con gran capacidad de adaptación. La presencia de dicha especie en Venezuela no está bien documentada y aún no se tiene certeza que sea un vector de Leishmania (Feliciangeli et al., 2006). Sin embargo, Oraá (2013) la reportó con infección natural en el estado Mérida.

Para la enfermedad, los modelos matemáticos de calentamiento global predicen un aumento dramático en la incidencia de leishmaniasis visceral y un ligero aumento en la incidencia de leishmaniasis cutánea (Fischer et al., 2011). Esto podría ser debido a diferencias en cómo Leishmania infantum Leishman y Donovan, 1903, reaccionaria a los aumentos de temperatura. Aunque P. perniciosus, vector de leishmaniasis visceral, disminuirá su abundancia, debido a efectos adversos de las altas temperaturas, en contraste se predice que la leishmaniasis visceral se incrementaría (Kuhn, 1999).

De los modelos bajo escenarios de cambio climático de las principales especies transmisoras de leishmaniasis cutánea en el estado Mérida, es importante resaltar el grado de solapamiento del espacio geográfico entre las principales especies vectoras en determinadas áreas, donde las condiciones serían las más favorables para su presencia, incluyendo nuevos espacios en áreas más pobladas y turísticas del estado Mérida, que implicarían un mayor riesgo de transmisión.

Es de esperar que si la temperatura ambiental, alcanza valores máximos, la transmisión podría cesar por completo, reduciendo los casos de leishmaniasis, ya que la capacidad vectorial podría aumentar significativamente debido al acortamiento del período de incubación y se daría una disminución en la supervivencia del vector (Benkova y Volf, 2007). Esto sugiere que los cambios de temperatura podrían afectar a la dinámica de desarrollo del insecto, de tal manera que se pudiera suprimir la transmisión si las condiciones ambientales se extienden más allá de la capacidad de supervivencia y adaptación (Bounoua et al., 2013). Sin embargo, los modelos de predicción a futuro bajo el efecto del cambio climático en el estado Mérida exacerban los riesgo de infección para leishmaniasis con expansión de las áreas de transmisión por la principal especie transmisora L. youngi y el solapamiento entre las principales especies en zonas más pobladas, como también es reportado para otras especies de flebotominos en otras latitudes (González et al., 2010).

Los modelos predictivos reflejan el efecto del cambio climático como una expansión en la transmisión y distribución de la leishmaniasis (Sutherst, 2001). Según Ready (2008), los modelos matemáticos no son concluyentes, sobre el efecto del cambio climático en la expansión de la leishmaniasis y se hacen necesarios más estudios, como investigar la adaptabilidad de los vectores a ese cambio climático. Un incremento en la temperatura y una disminución en la precipitación, pueden variar o crear las condiciones para la endemicidad de la leishmaniasis, si se encuentran dentro del rango óptimo para los vectores, hospedadores vertebrados y el parásito. La generación de los mapas de proyección sobre cambio climático de L. youngi, L. gomezi, L. ovallesi y L. walkeri, en el estado Mérida, permitió evidenciar cambios en la distribución futura de las principales especies de flebotominos en las zonas más pobladas del estado Mérida, lo que sugiere un mayor riesgo de transmisión de la leishmaniasis, principalmente en las áreas de solapamiento, información relevante para los entes de salud pública.

\section{AGRADECIMIENTOS}

Los autores agradecen la colaboración de todas las familias que apoyaron la realización de las capturas peridomiciliaria de los flebotominos, a la Universidad de Los Andes y al apoyo financiero del Proyecto Estratégico del FONACIT-Fondo Nacional de Ciencias, Tecnología e Innovación No 2011000371, bajo la coordinación de la Dra. Elsa Nieves.

\section{BIBLIOGRAFÍA}

Alexander, B. y Maroli, M. 2003. Control of phlebotomine sandflies. Medical and Veterinary Entomology 17: 1-18.

Alvar, J., Vélez, I., Bern, C., Herrero, M., Desjeux, P. y Cano, J. 2012. Leishmaniasis world wide and global estimates of its incidence. PLoS One 7(2):1-12. 
Anderson, R., Lew, D. y Peterson, A. 2003. Evaluating predictive models of species' distributions: criteria for selecting optimal models. Ecological Modelling 162:211-232.

Ataroff, M. y Sarmiento, L. 2004. Las unidades ecológicas de los Andes de Venezuela. En: La Marca, E., Soriano, P. (eds). Reptiles de Los Andes de Venezuela. Fundación Polar, Codepre-ULA, Fundacite-Mérida. Biogeos.

Bässler, C., Müller, J., Hothorn, T. y Kneib, T. 2009. Estimation of the extinction risk for high-montane species as a consequence of global warming and assessment of their suitability as cross-taxon indicators. Ecological Indicators. 10:341-352.

Benkova, I. y Volf, P. 2007. Effect of Temperature on Metabolism of Phlebotomus papatasi (Diptera: Psychodidae). Entomological Society of America 44:150-154.

Blanco, J. 2013. Modelos ecológicos: descripción, explicación y predicción. Ecosistemas 22(3):1-5.

Bounoua, L., Kahime, K., Houti, L., Blakey, T., Ebi, K., Zhang, P., Imhoff, M., Thome, K., Dudek, C., Sahabi, S., Messouli, M., Makhlouf, B., Laamrani, A. y Boumezzough, A. 2013. Linking climate to incidence of zoonotic cutaneous Leishmaniasis (L. major) in Pre-Saharan North Africa. International Journal of Environmental Research and Public Health 10:3172-91.

Cabaniel, G., Rada, L., Blanco, J., Rodríguez, A. y Escalera, J. 2005. Impacto de los eventos de El Niño Southern oscillation (ENSO) sobre la leishmaniosis cutánea en Sucre, Venezuela, a través del uso de información satelital, 1994 - 2003. Revista Peruana de Medicina Experimental y Salud Publica 22:32-38.

Campbell, D., Dujardin, J., Martínez, E., Feliciangeli, M., Pérez, J., Passerat de Silans, L. y Desjeux, P. 2001. Domestic and peridomestic transmission of American cutaneous leishmaniasis: changing epidemiological patterns present new control opportunities. Memórias do Instituto Oswaldo Cruz 96: 159-162.

Cárdenas, R., Sandoval, C., Rodríguez, A. y Franco, C. 2006. Impact of climate variability in the occurrence of leishmaniasis in northeastern Colombia. American Journal of Tropical Medicine and Hygiene 75:273-277.

70

Cross, E., Newcomb, W. y Tucker, C. 1996. Use of weather data and remote sensing to predict the geographic and seasonal distribution of Phlebotomus papatasi in southwest Asia. American Journal of Tropical Medicine and Hygiene 54:451-6.
Cross, E. y Hyams, K. 1996. The potential effect of global warming on the geographic and seasonal distribution of Phebotomus papatasi in southwest Asia. Environmental Health Perspectives 104:724-727.

Delgado, T. y Suarez, D. 2009. Efectos del cambio climático en la diversidad vegetal del corredor de conservación comunitaria Reserva Ecológica El Ángel- Bosque Protector Golondrinas en el norte del Ecuador. Ecología Aplicada 8(2):27-36.

Desjeux, P. 2001. The Increase in Risk Factors for the Leishmaniases Worldwide. Transactions Royal Society of Tropical Medicine and Hygiene 95(3):239-243.

Donalisio, M., Peterson, A., Costa, P., da Silva, F., Valencia, H., Shaw, J. y Filho, S. 2012. Microspatial Distributional Patterns of Vectors of Cutaneous Leishmaniasis in Pernambuco, Northeastern Brazil. Journal of Tropical Medicine 2012: 1-8.

Feliciangeli, M. 1987. Ecology of sandflies (Diptera:Psychodidae) in a restricted focus of cutaneous leishmaniasis in northern Venezuela. III. Seasonal fluctuation. Memórias do Instituto Oswaldo Cruz 82:167-176.

Fischer, D., Thomas, S. y Beierkuhnlein, C. 2011.Modelling climatic suitability and dispersal for disease vectors: the example of a phlebotomine sandfly in Europe. Procedia Environmental Sciencies 7:164-169.

Gaertner, M., Gutiérrez, J. y Castro, M. 2012. Escenarios regionales de cambio climático. Revista Española de Física 26(2):1-8.

González, C., Wang, O., Strutz, S., González, C., Sánchez, V. y Sarkar, S. 2010. Climate Change and Risk of Leishmaniasis in North America: Predictions from Ecological Niche Models of Vector and Reservoir Species. PLoS Neglected Tropical Diseases 4:1-15.

González, C., Paz, A. y Ferro, C. 2014. Predicted altitudinal shifts and reduced spatial distribution of Leishmania infantum vector species under climate change scenarios. Colombia. Acta Tropica 129(2014): 83-90.

Gordon, C., Cooper, C., Senior, C., Banks, H., Gregory, J., Johns, T., Mitchell, J. y Wood, R. 2000. The simulation of SST, sea ice extents and ocean heat transports in a version of the Hadley Centre coupled model without flux adjustments. Climate Dynamics 16: 147-168.

Graham, C., Moritz, C. y Williams, S. 2006 Habitat history improves prediction of biodiversity in rainforest fauna. Proceedings of the National Academy of Sciences 103: 632-636. 
Hartemink, N., Vanwambeke, S., Heesterbeek, H., Rogers, D., Morley, D., Pesson, B., Davies, C., Mahamdallie, S. y Ready, P. 2011. Integrated mapping of establishment risk for merging vector-borne infections: a case study of canine leishmaniasis in southwest France. PLoS One 6(8):1-12.

Hijmans, R., Cameron, S., Parra, J., Jones, P. y Jarvis, A. 2005a.Very high resolution interpolated climate surfaces for global land areas. International Journal of Climatology 25:1965-1978.

Hijmans, R., Guarino, L., Jarvis, A., O’Brien, R. y Mathur, P. 2005b. DIVA-GIS, versión 7.5. URL: http://www.diva-gis. org/. Consultado: 25 julio 2014.

Honty, G. 2011. Cambio climático: Negociaciones y consecuencias para América Latina. CLAES - Centro Latino Americano de Ecología Social, Uruguay.

Kigadye, E., Nkwengulila, G., Magesa, S., Abdulla, S. 2010. Diversity, spatial and temporal abundance of Anopheles gambiae complex in the Rufiji River basin, south-eastern Tanzania. Tanzania Journal of Health Research 12:68-72.

Kilpatrick, A., Fonseca, D., Ebel, G., Reddy, M. y Kramer, L. 2010. Spatial and temporal variation in vector competence of Culex pipiens and $C x$. restuans mosquitoes for West Nile virus. American Journal of Tropical Medicine and Hygiene 77:667-671.

Kuhn, K. 1999. Global warming and leishmaniasis in Italy. Bull. Tropical Medicine International Health 7:1-2.

Loiola, C., da Silva, D. y Galati, E. 2007. Phlebotomine fauna (Diptera: Psychodidae) and species abundance in an endemic area of American cutaneous leishmaniasis in southeastern Minas Gerais, Brazil. Memórias do Instituto Oswaldo Cruz 102(5):581-585.

López, R. y Molina, R. 2005. Cambio climático en España y riesgo de enfermedades infecciosas y parasitarias transmitidas por artrópodos y roedores. Revista Española de Salud Pública 79(2): 177-190.

Lugo, Y., Premoli, G. y Moreno, E. 1999. Detection of localized cutaneous leishmaniasis using conventional assay and Polymerase Chain Reaction: a report on three Venezuelan family groups. Boletín Dirección Malariología Saneamiento Ambiental 39:20-26.

Lugo, Y., Valera, M., Alarcón, M., Moreno, E., Premoli, G. y Colasante, C. 2003. Detección de Leishmania (Viannia) braziliensis en el endotelio vascular de lesiones de pacientes con leishmaniasis cutánea localizada. Investigación Clínica 44:61-76.
Magnuson, J. 2001. 150-year global ice record reveals major warming trend. International American Institute Global Change Research 24:22-25.

Maingon, R., Feliciangeli, D., Guzmán, B., Rodríguez, N., Convit, J., Adamson, R., Chance, M., Petralanda, I., Dougherty, M. y Ward, R. 1994. Cutaneous leishmaniasis in Táchira state, Venezuela. Annals Tropical Medical Parasitology 88:29-36.

Moo, D., Ibarra, C., Rebollar, E., Ibáñez, S., González, C. y Ramsey, J. 2013. Current and future niche of North and Central American sand flies (Diptera: Psychodidae) in climate change scenarios. PLoS Neglected Tropical Diseases 7(9): 1-13.

Moreno, E. y Scorza, J. 1998. Productos de excreción de Leishmania spp. de la región Andino - Venezolana. Revista de Ecología Latinoamericana Ambiental 5: 53-60.

Neuber, H. 2008. Leishmaniasis. Journal der Deutschen Dermatologischen Gesellschaft Impact Factor y Information 6: 754-765.

Nieves, E., Oraá, L., Rondón, Y., Sánchez, M., Sánchez, Y., Rujano, M., Rondón, M., Rojas, M., González, N. y Cazorla, D. 2014a. Riesgo de transmisión de Leishmania (Kinetoplastida: Trypanosomatidae) en Mérida Venezuela. Avances en Biomedicina 3: 57-64.

Nieves, E., Oraá, L., Rondón, Y., Sánchez, M., Sánchez, Y., Rojas, M., Rondón, M., Rujano, M., González, N. y Cazorla, D. 2014b. Effect of Environmental Disturbance on the Population of Sandflies and Leishmania Transmission in an Endemic Area of Venezuela. Journal of Tropical Medicine 2014:1-7.

Nieves, E., Oraá, L., Rondón, Y., Sánchez, M., Sánchez, Y., Rujano, M., Rondón, M., Rojas, M., Gonzalez, N. y Cazorla, D. 2015. Distribution of Vector Sandflies Leishmaniasis from an Endemic Area of Venezuela. Journal Tropical Diseases 3:1-7.

OMS. 2010. Control de la Leishmaniasis. Serie de Informes Técnicos 949. Ginebra, Suiza.

Oraá, L. 2013. Distribución, diversidad e influencia de variables ambientales y antropogénicas en las principales especies de flebotominos en Mérida Venezuela. Tesis de Grado. Universidad de Los Andes. Mérida, Venezuela.

Pabón, J. y Nicholls, R. 2005. El cambio climático y la salud humana. Biomédica 25(1):1-4. 
Parra, G. 2010. Sistemas de información geográfica y sensores remotos. Aplicaciones en enfermedades transmitidas por vectores. CES Medicina 24(2): 75-89.

Peterson, A. y Robins, C. 2003. Using ecological-niche modeling to predict barred owl invasions with implications for spotted owl conservation. Conservation Biology 17:11611165.

Peterson, A. y Shaw, J. 2003. Lutzomyia vectors for cutaneous leishmaniasis in Southern Brazil: ecological niche models, predicted geographic distributions, and climate change effects. International Journal Parasitology 33:919-931.

Peterson, A. 2006. Ecologic niche modeling and spatial patterns of disease transmission. Emerging Infectious Diseases 12:1822-1826.

Phillips, J., Anderson, P. y Schapire, E. 2006. Maximum entropy modeling of species geographic distributions. Ecological Modeling 190:231-259.

Phillips, J. y Dudik, M. 2008. Modeling of species distributions with MaxEnt: new extensions and a comprehensive evaluation. Ecography 31:161-175.

Pope, V., Gallani, M., Rowntree, P. y Stratton, R. 2000. The impact of new physical parametrizations in the Hadley Centre climate model: HadAM3. Climate Dynamics 16: 123-146.

Ready, P. 2008. Leishmaniasis emergence and climate change. Revue scientifique et technique 27(2):399-412.

Reiter, P. 2001. Climate change and mosquito borne disease. Environmental Health Perspectives 109(1): 141-161.

Rodríguez, N., Carrero, R., De Lima, H., Sandoval, I., Fernández, A. y Barrios, M. 2007. Impacto de Fenómenos Naturales (Deslaves y vaguadas) sobre la epidemiologia de la Leishmaniasis cutánea en zonas del estado Mérida. Salus 11:43-47.

Rodríguez, A., González, Y., Benítez, J., López, M., Harter, R., Vilca, L. y Cárdenas, R. 2010. Asociación entre la incidencia de leishmaniosis cutánea y el índice de desarrollo humano y sus componentes en cuatro estados endémicos de Venezuela. Revista Peruana de Medicina Experimental y Salud Pública 27:22-30.

Rondón, Y. 2015. Detección Parasitológica y Molecular de Leishmania en el Intestino de Flebotominos Vectores. Tesis de Grado. Universidad de Los Andes. Mérida Venezuela. 82p
Salomón, O., Rosa, J., Stein, M., Quintana, M., Fernández, M., Visintin, A., Spinelli, G., Bogado de Pascual, M., Molinari, M., Morán, M., Valdez, D. y Romero, M. 2008. Phlebotominae (Diptera: Psycodidae) fauna in the Chaco región and Cutaneous Leishmaniasis transmission patterns in Argentina. Memórias do Instituto Oswaldo Cruz 103(6): 578-584.

Sánchez, I., Liria, J. y Feliciangeli, M. 2015. Ecological Niche Modeling of Seventeen Sandflies Species (Diptera, Psychodidae, Phlebotominae) from Venezuela. International Journal of Zoology 2015:1-9.

Scheldeman, X., y Van Zonneveld, M. 2011. Manual de capacitación en análisis espacial de diversidad y distribución de plantas. Bioversity International.

Scorza, J., Valera, M., Moreno, E. y Jaimes, R. 1983. Epidemiologic survey of cutaneous leishmaniasis: an experience in Merida, Venezuela. Bulletin Panamerican Health Organ 7:361- 373.

Scorza, J., Castillo, L., Rezzano, S., Márquez, M. y Márquez, J. 1985. El papel del cafeto en la endemicidad de la leishmaniasis cutánea en Venezuela. Boletín Malariología Saneamiento Ambiental 25:82-88.

Shaw, J. 2007. The leishmaniases: survival and expansion in a changing world. A mini-review. Memórias do Instituto Oswaldo Cruz 102: 541-547.

Stott, P., Tett, S., Jones, G., Allen, M., Ingram, W. y Mitchell, J. 2001. Attribution of Twentieth Century Temperature Change to Natural and Anthropogenic Causes. Climate Dynamics 17:1-22.

Sutherst, R. 2001. Global change and human vulnerability to Vector-Born diseases. Clinical Microbiology Reviews 5:136-73.

Thomson, M., Elnaiem, D., Ashford, R. y Connor, S. 1999. Towards a kalaazar risk map for Sudan: mapping the potential distribution of Phlebotomus orientalis using digital data of environmental variables. Tropical Medicine International Health 4:105-113.

Valderrama, A., Tavares, M. y Andrade, J. 2011. Anthropogenic influence on the distribution, abundance and diversity of sandfly species (Diptera: Phlebotominae: Psychodidae), vectors of cutaneous Leishmaniasis in Panama. Memórias do Instituto Oswaldo Cruz 106(8):1024-1031.

Valera, M., Moreno, E. y Scorza, J. 1978. Cincuenta y seis casos de leishmaniasis tegumentaria en la cuenca de los ríos Chama - Mocotíes (Estado Mérida, Venezuela). Boletín Dirección Malariología Saneamiento Ambiental 28:238-247. 
Varela, S., Mateo, R., García, R. y Fernández, F. 2014. Macroecología y ecoinformática: sesgos, errores y predicciones en el modelado de distribuciones. Ecosistemas 23(1):46-53.

Vásquez, A., González, A., Góngora A., Prieto, E., Suárez, E. y Buitrago, L. 2013. Seasonal variation and natural infection of Lutzomyia antunesi (Diptera: Psychodidae: Phlebotominae), an endemic species in the Orinoquia region of Colombia. Memorias Instituto Oswaldo Cruz 108(4): 463-469.

Wang, X., Huang, X., Jiang, L. y Qiao, G. 2010. Predicting potential distribution of chestnut phylloxerid (Hemiptera:
Phylloxeridae) based on GARP and Maxent ecological niche models. Journal of Applied Entomology 134: 45-54.

Wisz, M., Hijmans, R., Li, J., Peterson, A., Graham, C. y Guisan, A. 2008. Predicting Species Distributions Working Group. Effects of sample size on the performance of species distribution models. Diversity and Distributions 14:763-773.

Young, D. y Duncan, M. 1994. Guide to the identification and geographic distribution of Lutzomyia sandflies in México, the West Indies, Central and South America (Diptera: Psychodidae). Memories of the American Entomological 54:779-881.

Fecha de recepción: 03/09/2015

Fecha de aceptación: 23/11/2015

Para citar este artículo: Nieves, E., M. Rujano, H. Ospino, L. Oraá, Y. Rondón, M. Sánchez, M. Rondón, Y. Sánchez, M. Rojas, N. González y D. Cazorla. 2015. Efectos del cambio climático sobre la distribución potencial de los Flebotominos transmisores de leishmaniasis en Mérida

Venezuela. Intropica Vol. 10: 60 - 73 